\title{
Nutrient composition and in vitro fermentability of corn grain and stover harvested at different periods in Goesan, a mountainous area
}

\author{
Kim Margarette Nogoy ${ }^{1}$, Yan Zhang ${ }^{1}$, Ye Hyun Lee ${ }^{1}$, Xiang Zi Li ${ }^{2}$, Hyun A Seong ${ }^{3^{*}}$ and Seong Ho Choi ${ }^{1^{*}}$ \\ ${ }^{1}$ Department of Animal Science, Chungbuk National University, Cheongju 28644, Korea \\ ${ }^{2}$ Co-Innovation Center of Beef Cattle Science and Industry Technology, Yanbian University, Yanji 133002, China \\ ${ }^{3}$ Department of Biochemistry, Chungbuk National University, Cheongju 28644, Korea
}

\begin{abstract}
With South Korea's limited capability of feed production because of its relatively small cultivable area, the country is pushed to depend on foreign feed imports despite the immensely fluctuating price of corn. Hence, intensive efforts to increase the total cultivable area in Korea like extending of farming to mountainous area is being practiced. Corn was planted in Goesan County, a mountainous area in the country. Grain and stover were harvested separately in three harvest periods: early-harvest (Aug 8), mid-harvest (Aug 18), and late-harvest (Aug 28). The nutrient composition such as dry matter (DM), crude protein $(C P)$, crude fat (EE), organic matter (OM), neutral detergent fiber (NDF), acid detergent fiber (ADF), and non-fibrous carbohydrates (NFC) was determined after harvest. Effective degradability (ED) of the major nutrients (DM, NDF, ADF, and CP) were measured through in vitro fermentation of rumen fluid from Hanwoo (Korean cattle). $\mathrm{pH}$, ammonia-N concentration, volatile fatty acid (VFA) concentration, and gas production were periodically measured at $0,3,6,12,24,48$, and $72 \mathrm{~h}$. Corn grain showed higher nutrient content and ED than stover. It also had higher gas production but its $\mathrm{pH}$, ammonia- $\mathrm{N}$, and total VFA concentration were lower than corn stover. The best nutrient composition of corn grain was observed in early-harvest (high $\mathrm{CP}, \mathrm{EE}, \mathrm{NDF}, \mathrm{OM}, \mathrm{NFC}$, and low ADF). Early-harvest of corn grain also had high effective degradability of dry matter (EDDM), effective degradability of neutral detergent fiber (EDNDF), effective degradability of acid detergent fiber (EDADF), and total VFA concentration. On the other hand, the best nutrient composition of stover was observed in mid-harvest (high $\mathrm{DM}, \mathrm{CP}, \mathrm{NDF}$, and low ADF). EDDM, EDNDF, and EDADF were pronounced in early-harvest and mid-harvest of stover but the latter showed high total VFA concentration. Hence, early and mid-harvested corn stover and grain in a mountainous area preserved their nutrients, which led to the effective degradation of major nutrients and high VFA production.
\end{abstract}

Keywords: Corn grain, Harvest, In vitro fermentation, Stover, Mountainous area

\section{Introduction}

Hanwoo (Korean cattle) population progressively increased to 2.6 million in 2015 from 1.8 million in 2005 [1] and to maintain its competitive quality, feeding of the cattle greatly depends on high energy corn-based diets. Corn is frequently used in in the finishing

Received: Dec 4, 2018 Revised: Jan 16,2019 Accepted: Jan 19,2019

"Corresponding author: Seong Ho Choi, Department of Animal Science, Chungbuk National University, Cheongju 28644, Korea.

Tel: +82-43-261-2544, E-mail: seongho@cbnu.ac.kr

Hyun A Seong, Depatment of Biochemistry, Chungbuk National University, Cheongju 28644, Korea

Tel: +82-43-261-2308, E-mail: haseung@cbnu.ac.kr

This is an Open Access article distributed under the terms of the Creative Commons Attribution Non-Commercial License (http://creativecommons.org/licenses/by$\mathrm{nc} / 4.0 /$ ) which permits unrestricted non-commercial use, distribution, and reproduction in any medium, provided the original work is properly cited.

Copyright (C) 2019 Korean Society of Animal Science and Technology. 
phase because it increases carcass quality grades by increasing fat deposition (especially intramuscular) [1], which results in a more desirable product for consumers. Aside from it, corn is used in beef cattle feeding because of its advantages in improving the efficiency of growth [2]. However, corn production in South Korea is negligible because it accounts for less than one percent of total cultivation. With the country's limited capability of feed production because of its relatively small cultivable area, the country is pushed to depend on foreign feed imports despite the fact that the price of corn has been fluctuating immensely. Hence, intensive efforts to increase the total cultivable area in Korea like extending of farming to mountainous area is being practiced.

As with all the corn variety, its nutritive value is determined by the nutrient composition of the relative proportions of the different plant fractions (stover and ear) and their degradability. The ear or grain of the corn plant contains starch which accounts for about $70 \%$ of the total dry matter (DM) of the grain itself and is considered one of its most digestible components while the corn stover (stalk, leaf, husk, and cob) represents approximately $70 \%$ of the whole plant DM. Most of the studies concentrated on grain production because it was thought for a long time that a greater quantity of grain would give a greater portion of starch and therefore, a more digestible DM [3]. Until quite recently, some studies reported that in addition to grain, corn stover plays a role in improving the quality of feed [4-5] aside from its potential on reducing feed cost. Nutrient composition of these plant fractions are greatly influenced by the stage of maturity at which they are harvested, but the influence of harvest period on in vitro fermentability, a knowledge of which could improve the feed efficiency of corn in ruminant animals, is not always understood. As reported by Preston [6], the efficiency of forage in the rumen ecosystem cannot be determined by simply doing a conventional feed analysis.

The study aimed to determine the nutrient composition, and in vitro fermentability of corn grain and stover harvested at different periods in mountainous area. In vitro fermentability includes and is limited to determination of $\mathrm{pH}$ values, total gas production, ammonia-N concentration, VFA proportions, and effective degradability of major nutrient components (DM, NDF, and ADF).

\section{Materials and Methods}

\section{Sample collection}

Cultivation of Gwangpyung-ok, a domestic variety of corn in Korea was carried out in $16,945 \mathrm{~m}^{2}$ land in Goesan, North Chungcheong, South Korea. Meteorological Agency of Goesan monitored local weather during the cultivation period. The average temperature of the area during the conduct of the study ranged from $18.8^{\circ} \mathrm{C}$ (May) to $26.8^{\circ} \mathrm{C}$ (Aug) while average precipitation ranged from $38.9 \mathrm{~mm}$ (Jun) to $374.4 \mathrm{~mm}$ (Jul). Soil analyses such as organic matter, $\mathrm{pH}$, and soil electric conductivity were $21 \mathrm{~g} / \mathrm{kg}$, 6.1, $1.7(\mathrm{dS} / \mathrm{m})$, respectively. Corn was sown on May 28, 2015, and grain and stover were harvested on three different periods: Aug 8 (early-harvest), Aug 18 (mid-harvest), and Aug 28 (late-harvest). Samples were weighed and dried at $65^{\circ} \mathrm{C}$ for $72 \mathrm{~h}$ thru forced-air drying oven and then ground through a Wiley mill using $1 \mathrm{~mm}$ screen before analysis.

\section{Nutrient composition analyses}

The nutrient composition of corn grain and corn stover were analyzed as follows: crude protein $(\mathrm{CP})$ calculated as $\mathrm{N} \times 6.25$, ether extract (EE), and ash were based on the AOAC [7] method; neutral detergent fiber (NDF) and acid detergent fiber (ADF) were accorded to Van Soest et al. [8].

\section{In vitro incubation}

Rumen contents were obtained $2 \mathrm{~h}$ after the morning feeding (08:00) from two ruminal-cannulated non-lactating Korean native cows (Hanwoo) fed $9 \mathrm{~kg} / \mathrm{d}$ total diets daily $(2 \mathrm{~kg}$ concentrate and $7 \mathrm{~kg}$ ryegrass, as fed basis), twice per day in an equal volume. The rumen fluid was hand-squeezed and filtered through 8 layers of cheesecloth and kept in a water bath at $39^{\circ} \mathrm{C}$. Incubation solution was prepared by mixing $50 \mathrm{~mL}$ filtered rumen fluid with $100 \mathrm{~mL}$ McDougall's artificial saliva [9] in a $250 \mathrm{~mL}$ Erlenmeyer flask. One nylon bag contained one gram of dried and ground corn grain and stover samples (weight per surface area: $40 \mathrm{mg} / \mathrm{cm}^{2}$ ) and was incubated in the flasks for 3, 6, 12, 24, 48, and $72 \mathrm{~h}$. The flasks were then sealed with silicone rubber stoppers with a 3-way stopcock and were incubated anaerobically in an orbital shaking incubator at a speed of $135 \mathrm{rpm}$ up to $24 \mathrm{~h}$ at $39^{\circ} \mathrm{C}$. Carbon dioxide $\left(\mathrm{CO}_{2}\right)$ was continuously flushed all throughout the operations. The in vitro incubation was conducted 3 times with each treatment in duplicate each time under similar conditions.

Incubation was stopped by removing the bottles from the shaking incubator at 3, 6, 12, 24, 48, and $72 \mathrm{~h}$. Gas was read by inserting calibrated glass syringe into the 3 -way stopcock and the $\mathrm{pH}$ of the incubated solution was also immediately measured. At the same time, an aliquot of incubated solution (two $1 \mathrm{~mL}$ ) was collected from each flask for ammonia and volatile fatty acid (VFA) analysis. The $1 \mathrm{~mL}$ aliquot for ammonia analysis was mixed with $0.2 \mathrm{~mL}$ phosphoric acid to stop the fermentation. Ammonia concentration was determined using a spectrophotometer through the method of Fawcett and Scott [10]. The other $1 \mathrm{~mL}$ aliquot was mixed with the $0.02 \mathrm{~mL}$ pivalic acid solution as the internal standard for the VFA analysis as described by Li et al. [11]. After incubation, the nylon bags were removed from the flasks, washed in tap water till the rinse solution became clear, and then dried at 
$80^{\circ} \mathrm{C}$ for $48 \mathrm{~h}$ in the drying oven to measure DM, NDF, and ADF degradation.

Percent DM, NDF, ADF disappearance at each incubation time were calculated from the portion remaining after incubation, and the rate was fitted to the equation of Orskov and McDonald [12] as follows:

$$
Y(t)=a+b\left(1-e^{c t}\right) \text { where, }
$$

$\mathrm{Y}(\mathrm{t})=$ proportion of the incubated material degraded at time $\mathrm{t}$; $\mathrm{t}=$ incubation time $(\mathrm{h}) ; \mathrm{a}=$ highly soluble and instantly degradable fraction; $b=$ insoluble and slowly degradable fraction; $c=$ rate constant of degradation $\left(\% \mathrm{~h}^{-1}\right)$; and $\mathrm{e}=2.7182$ (base for natural logarithm). Parameters $a, b$, and $c$ were estimated by the Marquardt iterative procedure using the PROC NLIN of SAS [12]. The effective degradability of DM (EDDM), NDF (EDNDF), and $\mathrm{ADF}(\mathrm{EDADF})$ of corn grain and corn stover were calculated using the following equation [12]:

$$
\mathrm{ED}=\mathrm{a}+(\mathrm{b} \times \mathrm{c}) /(\mathrm{c}+\mathrm{r}) \text { where }
$$

$r=$ rate constant of passage $\left(\% \mathrm{~h}^{-1}\right)$ and a hypothetical of $0.05 /$ $\mathrm{h}$ and $0.023 / \mathrm{h}$ were used for estimation for corn grain and stover, respectively.

\section{Statistical analyses}

The study was conducted as a $2 \times 3$ factorial design representing two fractions of corn (corn grain and corn stover) and three harvesting periods (early, mid, and late). Six treatments were replicated twice per time and repeated three times. For each variable measured at each time, replicates were averaged, and the total number of observations was 6 (treatments) $\times 3$ (times) $=18$ observations. The 18 observations were subjected to least square analysis of variance ANOVA by using the GLM procedure in SAS [13]. Significances were declared at $p<0.05$ and if differences were detected, data were further subjected to Duncan's multiple range test (DMRT).

\section{Results}

Nutrient composition of corn stover and grain were significantly affected by harvest period, plant fractions, and their interactions (Table 1). DM, CP, EE, OM (organic matter), and NFC (neutral detergent fiber) contents were higher in corn grain than in stover $(p<0.001)$ while NDF and ADF contents were higher in stover than in grain $(p<0.001)$. DM content was high in the mid and late-harvest of both plant fractions $(p<0.05)$. CP content of early-harvest corn grain was ranked highest followed by mid-harvest of stover $>$ late-harvest grain $>$ late-harvest stover $>$ mid-harvest grain $>$ early-harvest stover $(p<0.001)$. EE content was high in early-harvest of both fractions $(p<0.01)$. NDF content was high in mid and late-harvest of stover followed by early-harvest stover $>$ early-harvest corn grain > mid-harvest corn grain > late-harvest corn grain $(p<0.001)$. On the other hand, ADF content was high in late-harvest of both plant fractions $(p<0.001)$ and OM content was high in early and late-harvest regardless of plant fractions $(p<0.01)$. Lastly, NFC was highest in late-harvest of corn grain followed by early-harvest grain $>$ mid-harvest grain $>$ late-harvest stover $>$ early-harvest stover $>$ mid-harvest stover $(p<0.001)$.

Degradation parameters ( $a, b$, and $c)$ and effective degradability of the major nutrients (DM, NDF, $\mathrm{ADF}$, and $\mathrm{CP}$ ) were presented in Table 2. EDNDF and EDADF were not significantly affected by plant fractions but EDDM was found higher in corn grain, and stover had higher EDCP $(p<0.001)$. In terms of the harvest period, increased EDDM was found in early and mid-harvest of corn grain followed by late-harvest corn grain > early and mid-harvest

\begin{tabular}{|c|c|c|c|c|c|c|c|c|c|c|}
\hline \multirow{2}{*}{$\begin{array}{c}\text { Nutrient } \\
\text { composition }\end{array}$} & \multicolumn{3}{|c|}{ Corn stover } & \multicolumn{3}{|c|}{ Corn grain } & \multirow{2}{*}{ SEM } & \multicolumn{3}{|c|}{ Effects } \\
\hline & Early & Mid & Late & Early & Mid & Late & & Harvest (H) & Fraction (F) & $H \times F$ \\
\hline $\mathrm{DM}, \%$ & $92.77^{\mathrm{d}}$ & $93.45^{\mathrm{b}}$ & $94.23^{b}$ & $93.69^{c}$ & $94.31^{a}$ & $94.28^{a}$ & 0.27 & * & $* * *$ & $* * *$ \\
\hline $\mathrm{CP}, \%$ & $7.44^{f}$ & $8.35^{\mathrm{b}}$ & $7.69^{d}$ & $9.10^{a}$ & $7.43^{\mathrm{e}}$ & $7.81^{\mathrm{c}}$ & 0.24 & $* * *$ & $* *$ & $* * *$ \\
\hline EE, \% & $1.52^{\mathrm{c}}$ & $1.34^{\mathrm{d}}$ & $1.34^{\mathrm{d}}$ & $6.25^{\mathrm{a}}$ & $5.01^{\mathrm{b}}$ & $4.64^{\mathrm{b}}$ & 0.36 & $* *$ & $* \star *$ & $* \star$ \\
\hline NDF, \% & $67.77^{\mathrm{b}}$ & $68.88^{a}$ & $68.57^{\mathrm{a}}$ & $26.92^{\mathrm{c}}$ & $27.87^{\mathrm{c}}$ & $25.70^{d}$ & 0.45 & $* * *$ & $* * *$ & $* *$ \\
\hline$A D F, \%$ & $37.78^{\mathrm{b}}$ & $39.56^{\mathrm{b}}$ & $39.70^{\mathrm{a}}$ & $10.01^{d}$ & $11.06^{\mathrm{d}}$ & $11.82^{c}$ & 0.47 & $* * *$ & $* \star *$ & *** \\
\hline $\mathrm{OM}, \%$ & $93.54^{c}$ & $92.06^{d}$ & $93.88^{c}$ & $98.74^{a}$ & $97.99^{b}$ & $98.90^{\mathrm{a}}$ & 0.32 & $* *$ & $* * *$ & $* * *$ \\
\hline NFC, $\%$ & $16.82^{\mathrm{e}}$ & $12.49^{f}$ & $16.28^{d}$ & $57.47^{\mathrm{b}}$ & $57.68^{\mathrm{c}}$ & $60.75^{\mathrm{a}}$ & 0.66 & $* * *$ & $* \star \star$ & $\star \star \star *$ \\
\hline
\end{tabular}

Table 1. Nutrient composition of corn stover and grain harvested at different periods

${ }^{a-f}$ Means in the same row with different superscripts are significantly different.

${ }^{*} p<0.05,{ }^{* *} p<0.01,{ }^{* * *} p<0.001$.

SEM, standard error of means; NS, not significant; NFC was calculated by difference: [100-(NDF, \% + crude protein, $\%+$ fat, $\%+$ ash, $\%)]$; DM, dry matter; CP, crude protein; EE, ether extract (crude fat); NDF, neutral detergent fiber; ADF, acid detergent fiber; OM, organic matter; NFC, non-fibrous carbohydrates. 
Table 2. Effective degradation of major nutrient composition (DM, NDF, ADF, CP) and degradation parameters (a,b,c) of corn stover and grain harvested at different periods

\begin{tabular}{|c|c|c|c|c|c|c|c|c|c|c|}
\hline & \multicolumn{3}{|c|}{ Corn stover } & \multicolumn{3}{|c|}{ Corn grain } & \multirow{2}{*}{ SEM } & \multicolumn{3}{|c|}{ Effects } \\
\hline & Early & Mid & Late & Early & Mid & Late & & Harvest (H) & Fraction (F) & $\mathrm{H} \times \mathrm{F}$ \\
\hline a & $25.44^{\mathrm{D}}$ & $23.77^{\mathrm{E}}$ & $18.39^{F}$ & $40.84^{\mathrm{A}}$ & $39.60^{B}$ & $37.28^{C}$ & 1.28 & $* * *$ & $* * *$ & ** \\
\hline$b$ & $17.96^{\mathrm{D}}$ & $19.89^{\mathrm{D}}$ & $29.67^{C}$ & $36.26^{\mathrm{B}}$ & $34.17^{B}$ & $35.46^{A}$ & 4.09 & ** & $* \star *$ & ** \\
\hline c & $0.03^{\mathrm{BC}}$ & $0.05^{\mathrm{A}}$ & $0.02^{C}$ & $0.04^{\mathrm{BC}}$ & $0.06^{A}$ & $0.04^{c}$ & 0.00 & * & NS & NS \\
\hline EDDM & $35.53^{C}$ & $37.05^{\mathrm{c}}$ & $32.01^{\mathrm{D}}$ & $57.13^{A}$ & $57.02^{\mathrm{A}}$ & $53.00^{\mathrm{B}}$ & 3.44 & $* *$ & $* * *$ & NS \\
\hline a & $8.88^{A}$ & $4.59^{B}$ & $3.4^{\mathrm{C}}$ & $6.23^{B}$ & $7.65^{A}$ & $2.14^{\mathrm{C}}$ & 1.29 & $* * *$ & NS & *** \\
\hline$b$ & $20.71^{\mathrm{A}}$ & $22.19^{B}$ & $19.59^{B}$ & $39.00^{\mathrm{A}}$ & $19.58^{\mathrm{B}}$ & $24.24^{B}$ & 4.78 & $* \star *$ & $* * *$ & $* * *$ \\
\hline c & $0.03^{B}$ & $0.03^{A}$ & $0.05^{A}$ & $0.02^{\mathrm{B}}$ & $0.06^{A}$ & $0.05^{\mathrm{A}}$ & 0.00 & $* * *$ & NS & * \\
\hline EDNDF & $20.17^{\mathrm{A}}$ & $17.10^{\mathrm{AB}}$ & $16.55^{\mathrm{B}}$ & $17.24^{\mathrm{A}}$ & $17.92^{\mathrm{AB}}$ & $14.34^{\mathrm{B}}$ & 3.19 & * & NS & NS \\
\hline a & $11.70^{\mathrm{A}}$ & $3.89^{\mathrm{B}}$ & $3.49^{B}$ & $13.31^{\mathrm{A}}$ & $6.12^{B}$ & $1.83^{\mathrm{B}}$ & 1.64 & $* * *$ & NS & $* *$ \\
\hline b & 16.38 & 20.82 & 14.75 & 11.01 & 11.41 & 16.47 & 4.06 & NS & ** & ** \\
\hline c & $0.03^{C}$ & $0.03^{\mathrm{BC}}$ & $0.09^{B}$ & $0.08^{B}$ & $0.13^{\mathrm{AB}}$ & $0.08^{A}$ & 0.03 & * & $* * *$ & $* * *$ \\
\hline EDADF & $20.12^{\mathrm{A}}$ & $14.34^{\mathrm{B}}$ & $14.97^{\mathrm{B}}$ & $19.88^{\mathrm{A}}$ & $14.19^{B}$ & $11.99^{B}$ & 2.96 & $* * *$ & NS & NS \\
\hline$a$ & $53.43^{C}$ & $60.49^{B}$ & $66.16^{A}$ & $28.65^{F}$ & $39.69^{E}$ & $40.52^{D}$ & 1.15 & $* * *$ & $* * *$ & $* * *$ \\
\hline b & $18.65^{\mathrm{C}}$ & $16.20^{\mathrm{D}}$ & $13.24^{\mathrm{D}}$ & $55.66^{A}$ & $33.24^{B}$ & $33.00^{B}$ & 3.11 & $* * *$ & $* * *$ & $* * *$ \\
\hline c & $0.05^{\mathrm{AB}}$ & $0.03^{B}$ & $0.06^{A}$ & $0.02^{\mathrm{CD}}$ & $0.01^{\mathrm{D}}$ & $0.02^{c}$ & 0.00 & * & $\star * * *$ & NS \\
\hline EDCP & $65.48^{C}$ & $69.06^{\mathrm{B}}$ & $75.32^{\mathrm{A}}$ & $43.35^{\mathrm{F}}$ & $46.53^{\mathrm{E}}$ & $50.43^{\mathrm{D}}$ & 2.38 & $* \star *$ & $* \star *$ & NS \\
\hline
\end{tabular}

\footnotetext{
${ }^{A-F}$ Means in the same row with different superscripts are significantly different.
}

${ }^{*} p<0.05,{ }^{* *} p<0.01,{ }^{* * *} p<0.001$.

DM, dry matter; NDF, neutral detergent fiber; ADF, acid detergent fiber, CP, crude protein; SEM, standard error of means, NS, not significant; EDDM, effective degradability of dry matter; EDNDF, effective degradability of neutral detergent fiber; EDADF, effective degradability of acid detergent fiber; EDCP, effective degradability of crude protein; a, b, $\mathrm{c}$ in first column corresponds to: a, highly soluble fraction of sample; b, insoluble and slowly soluble fraction of sample at time infinity; c, rate constant of degradation of fraction "b."

stover $>$ late-harvest stover $(p<0.01)$. EDNDF $(p<0.05)$ and $\mathrm{EDADF}(p<0.001)$ were highest in early-harvest in both corn grain and stover. EDCP of late-harvest stover ranked highest followed by mid-harvest $>$ early-harvest $>$ late-harvest corn grain > mid-harvest corn grain > early-harvest corn grain $(p<0.001)$.

The concentration of total VFA produced and VFA molar proportions were shown in Table 3. At all incubation time except at $6 \mathrm{~h}$, total VFA concentration was significantly affected by harvest period, plant fractions, and their interactions. Total VFA concentration of stover was higher than corn grain $(p<0.001)$ at all incubation time. At $3 \mathrm{~h}$, total VFA was highest in late-harvest stover but at 12 to $72 \mathrm{~h}$, total VFA was consistently highest in mid-harvest stover followed by late and early-harvest $(p<0.001)$. For corn grain, early-harvest had the highest total VFA concentration at $3 \mathrm{~h}(p<$ $0.001), 12 \mathrm{~h}(p<0.05)$, and $72 \mathrm{~h}(p<0.001)$. Molar proportions of acetate and propionate and their ratio were significantly affected by plant fractions from $0 \mathrm{~h}$ to $72 \mathrm{~h}(p<0.001)$ and harvest period at $3-12 \mathrm{~h}$ incubation time $(p<0.05) . \mathrm{C}_{2} / \mathrm{C}_{3}$ ratio at $3-12 \mathrm{~h}$ was consistently highest in early-harvest stover $(p<0.01)$. Valerate proportion was also significantly affected by plant fractions from 0 to $72 \mathrm{~h}(p<0.001)$ and harvest period at $3-12 \mathrm{~h}$ incubation time $(p<$ 0.05). Total gas production (Fig. 1) of all treatments increased with incubation time. Total gas produced at 12,24 , and $72 \mathrm{~h}$ incubation time was higher in corn grain fraction than the stover $(p<0.01)$, but no significant effect was found on harvest period and their interactions. As shown in Fig. 2, the ammonia- $\mathrm{N}$ concentration of all treatments increased with incubation time. At 0 to $72 \mathrm{~h}$, corn stover had higher ammonia- $\mathrm{N}$ concentration than grain $(p<0.001)$ and no significant effect was found in harvest period and their interactions. Meanwhile, $\mathrm{pH}$ of all treatments (Fig. 3) decreased with incubation time. No significant effect was found in harvest period at all incubation time but plant fractions showed a significant effect. Corn grain had higher $\mathrm{pH}$ at $0 \mathrm{~h}(p<0.05)$ but at $6-72 \mathrm{~h}$ incubation time, $\mathrm{pH}$ values were lower than that of the corn stover $(p<0.001)$.

\section{Discussion}

Nutritive value of corn is determined by its nutrient composition, as well as its effective degradation. It varies significantly depending upon the plant fractions and stage of maturity at harvest. Nutrient composition of corn plant cultivated in Goesan, a mountainous area, varied as affected by plant fractions and different harvest period. Higher DM, CP, EE, OM, and NFC contents were found 
Table 3. Total volatile fatty acids (VFA) concentration and VFA molar proportions of corn stover and grain harvested at different periods

\begin{tabular}{|c|c|c|c|c|c|c|c|c|c|c|c|}
\hline & & \multicolumn{3}{|c|}{ Corn stover } & \multicolumn{3}{|c|}{ Corn grain } & \multirow{2}{*}{ SEM } & \multicolumn{3}{|c|}{$p$-values } \\
\hline & & Early & Mid & Late & Early & Mid & Late & & Harvest & tion (F) & $H \times F$ \\
\hline & & \multicolumn{10}{|c|}{$3 \mathrm{~h}$} \\
\hline \multicolumn{2}{|c|}{ Total VFA (mmoles/100 mL) } & $55.56^{\mathrm{b}}$ & $56.11^{b}$ & $86.48^{a}$ & $38.97^{c}$ & $32.94^{d}$ & $35.55^{\mathrm{cd}}$ & 3.31 & $* * *$ & $* * *$ & $* * *$ \\
\hline \multirow{6}{*}{$\begin{array}{l}\text { Molar proportion } \\
\text { (mmoles/100 mmoles) }\end{array}$} & $\mathrm{C}_{2}$ & $66.50^{\mathrm{ab}}$ & $68.18^{\mathrm{a}}$ & $66.74^{\mathrm{b}}$ & $61.83^{\mathrm{c}}$ & $60.98^{d}$ & $60.32^{d}$ & 0.65 & * & $* * *$ & $* *$ \\
\hline & $\mathrm{C}_{3}$ & $21.80^{\mathrm{c}}$ & $19.22^{\mathrm{c}}$ & $23.12^{b}$ & $21.62^{b}$ & $24.36^{\mathrm{b}}$ & $25.43^{\mathrm{a}}$ & 0.81 & $* * *$ & 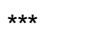 & $* * *$ \\
\hline & $\mathrm{C}_{4}$ & $8.78^{c}$ & $8.66^{c}$ & $7.56^{d}$ & $10.94^{a}$ & $10.50^{a}$ & $10.34^{\mathrm{b}}$ & 0.34 & $* * *$ & *** & * \\
\hline & $\mathrm{C}_{5}$ & $0.84^{\mathrm{b}}$ & $1.07^{b}$ & $0.74^{\mathrm{C}}$ & $1.65^{\mathrm{a}}$ & $1.12^{\mathrm{a}}$ & $1.04^{\mathrm{b}}$ & 0.15 & $* * *$ & *** & $* * *$ \\
\hline & $\mathrm{C}_{2} / \mathrm{C}_{3}$ & $3.06^{\mathrm{a}}$ & $3.56^{\mathrm{a}}$ & $2.89^{b}$ & $2.86^{b}$ & $2.51^{\mathrm{b}}$ & $2.37^{c}$ & 0.13 & $* * *$ & *** & $* * *$ \\
\hline & & \multicolumn{10}{|c|}{$6 \mathrm{~h}$} \\
\hline \multicolumn{2}{|c|}{ Total VFA (mmoles/100 mL) } & 65.62 & 67.52 & 71.28 & 40.65 & 41.26 & 48.46 & 8.58 & NS & *** & NS \\
\hline \multirow{6}{*}{$\begin{array}{l}\text { Molar proportion } \\
\text { (mmoles/100 mmoles) }\end{array}$} & $\mathrm{C}_{2}$ & 65.81 & 69.04 & 64.11 & 60.97 & 59.69 & 61.54 & 1.62 & NS & *** & $* *$ \\
\hline & $\mathrm{C}_{3}$ & $21.95^{\mathrm{b}}$ & $20.12^{\mathrm{b}}$ & $24.71^{a}$ & $22.92^{\mathrm{b}}$ & $25.65^{a}$ & $25.1^{a}$ & 1.51 & $* *$ & $* *$ & ** \\
\hline & $\mathrm{C}_{4}$ & $9.17^{b}$ & $7.62^{c}$ & $8.42^{\mathrm{c}}$ & $11.30^{\mathrm{a}}$ & $10.66^{\mathrm{b}}$ & $9.82^{\mathrm{b}}$ & 0.48 & $* \star *$ & *** & ** \\
\hline & $\mathrm{C}_{5}$ & $0.85^{c}$ & $0.87^{c}$ & $0.81^{d}$ & $1.44^{\mathrm{a}}$ & $1.15^{\mathrm{ab}}$ & $0.99^{b}$ & 0.56 & * & 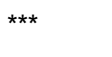 & NS \\
\hline & $\mathrm{C}_{2} / \mathrm{C}_{3}$ & $3.00^{\mathrm{a}}$ & $3.44^{\mathrm{a}}$ & $2.60^{\mathrm{b}}$ & $2.66^{b}$ & $2.35^{\mathrm{C}}$ & $2.46^{\mathrm{c}}$ & 0.67 & ** & *** & $* * *$ \\
\hline & & \multicolumn{10}{|c|}{$12 \mathrm{~h}$} \\
\hline \multicolumn{2}{|c|}{ Total VFA (mmoles/100 mL) } & $84.01^{\mathrm{b}}$ & $112.26^{a}$ & $103.34^{\mathrm{ab}}$ & $51.62^{c}$ & $49.94^{d}$ & $49.29^{d}$ & 10.16 & * & $* * *$ & * \\
\hline \multirow{6}{*}{$\begin{array}{l}\text { Molar proportion } \\
\text { (mmoles/100 mmoles) }\end{array}$} & $\mathrm{C}_{2}$ & $64.97^{\mathrm{b}}$ & $70.66^{a}$ & $65.51^{\mathrm{b}}$ & $59.25^{c}$ & $57.67^{d}$ & $57.97^{d}$ & 1.63 & * & $* * *$ & $* * *$ \\
\hline & $\mathrm{C}_{3}$ & $22.13^{d}$ & $19.25^{d}$ & $23.03^{c}$ & $25.04^{b}$ & $26.59^{b}$ & $27.32^{\mathrm{a}}$ & 1.6 & * & $\star \star \star *$ & * \\
\hline & $\mathrm{C}_{4}$ & 9.74 & 7.38 & 8.5 & 11.58 & 12.41 & 11.36 & 0.96 & NS & $* * *$ & * \\
\hline & $\mathrm{C}_{5}$ & $0.93^{b}$ & $0.75^{c}$ & $0.87^{c}$ & $1.21^{\mathrm{a}}$ & $0.93^{b}$ & $0.99^{b}$ & 0.14 & * & ** & NS \\
\hline & $\mathrm{C}_{2} / \mathrm{C}_{3}$ & $2.94^{a}$ & $3.68^{b}$ & $2.86^{\mathrm{a}}$ & $2.37^{c}$ & $2.18^{d}$ & $2.14^{d}$ & 0.21 & ** & $\star \star \star ~$ & $\star \star \star *$ \\
\hline & & \multicolumn{10}{|c|}{$24 \mathrm{~h}$} \\
\hline \multicolumn{2}{|c|}{ Total VFA (mmoles/100 mL) } & $86.82^{b}$ & $158.81^{a}$ & $137.72^{a}$ & $60.64^{d}$ & $57.89^{d}$ & $69.34^{\mathrm{c}}$ & 7.4 & $* * *$ & *** & $* * *$ \\
\hline \multirow{6}{*}{$\begin{array}{l}\text { Molar proportion } \\
\text { (mmoles/100 mmoles) }\end{array}$} & $\mathrm{C}_{2}$ & 63.74 & 66.54 & 68.12 & 56.37 & 54.73 & 53.51 & 2.48 & NS & $* * *$ & * \\
\hline & $\mathrm{C}_{3}$ & 24.14 & 24.2 & 22.53 & 26.97 & 28.19 & 29.79 & 1.65 & NS & $* * *$ & * \\
\hline & $\mathrm{C}_{4}$ & 9.06 & 6.81 & 6.84 & 12.94 & 13.21 & 13.52 & 1.18 & NS & $\star \star \star ~$ & * \\
\hline & $\mathrm{C}_{5}$ & 0.91 & 0.78 & 0.81 & 1.13 & 1.18 & 1.01 & 0.35 & NS & *** & NS \\
\hline & $\mathrm{C}_{2} / \mathrm{C}_{3}$ & 2.65 & 2.75 & 3.04 & 2.09 & 1.95 & 1.81 & 0.22 & NS & $* * *$ & * \\
\hline & & \multicolumn{10}{|c|}{$48 \mathrm{~h}$} \\
\hline \multicolumn{2}{|c|}{ Total VFA (mmoles/100 mL) } & $96.28^{\mathrm{c}}$ & $159.90^{\mathrm{a}}$ & $124.00^{b}$ & $76.51^{f}$ & $90.56^{d}$ & $83.99^{e}$ & 9 & $* \star *$ & $* * *$ & $* \star *$ \\
\hline \multirow{6}{*}{$\begin{array}{l}\text { Molar proportion } \\
\text { (mmoles/100 mmoles) }\end{array}$} & $\mathrm{C}_{2}$ & 63.78 & 67.33 & 66.16 & 51.85 & 52.93 & 54.23 & 2.36 & NS & *** & NS \\
\hline & $\mathrm{C}_{3}$ & 24.55 & 23.84 & 23.3 & 28.52 & 29.51 & 28.7 & 2.1 & NS & *** & NS \\
\hline & $\mathrm{C}_{4}$ & $8.6^{\mathrm{c}}$ & $6.47^{\mathrm{d}}$ & $7.78^{\text {cd }}$ & $14.8^{\mathrm{a}}$ & $13.23^{\mathrm{ab}}$ & $13.47^{\mathrm{ab}}$ & 1.31 & * & *** & NS \\
\hline & $\mathrm{C}_{5}$ & $0.91^{\mathrm{c}}$ & $0.73^{d}$ & $0.83^{\mathrm{c}}$ & $2.04^{\mathrm{a}}$ & $1.87^{\mathrm{a}}$ & $1.56^{\mathrm{b}}$ & 0.18 & * & *** & * \\
\hline & $\mathrm{C}_{2} / \mathrm{C}_{3}$ & 2.61 & 2.23 & 2.91 & 1.82 & 1.82 & 1.83 & 0.33 & NS & *** & NS \\
\hline & & \multicolumn{10}{|c|}{$72 \mathrm{~h}$} \\
\hline \multicolumn{2}{|c|}{ Total VFA (mmoles/100 mL) } & $104.24^{c}$ & $162.14^{\mathrm{a}}$ & $131.87^{b}$ & $93.56^{d}$ & $89.78^{e}$ & $84.49^{f}$ & 2.85 & $\star * *$ & *** & $* \star *$ \\
\hline $\begin{array}{l}\text { Molar proportion } \\
\text { (mmoles } / 100 \text { mmoles })\end{array}$ & $\mathrm{C}_{2}$ & 62.89 & 64.29 & 63.47 & 50.81 & 50.88 & 52.4 & 1.98 & NS & $* * *$ & NS \\
\hline & $\mathrm{C}_{3}$ & 24.6 & 26.54 & 25.46 & 28.59 & 27.45 & 26.62 & 1.72 & NS & $* *$ & NS \\
\hline & $\mathrm{C}_{4}$ & 8.82 & 6.7 & 7.97 & 14.24 & 14.62 & 15.07 & 1.3 & NS & $\star * \star *$ & NS \\
\hline & $\mathrm{C}_{5}$ & 1.23 & 0.8 & 1.03 & 3.31 & 3.33 & 2.72 & 0.35 & NS & $\star \star \star *$ & NS \\
\hline & $\mathrm{C}_{2} / \mathrm{C}_{3}$ & 2.56 & 2.42 & 2.51 & 1.78 & 1.87 & 1.98 & 0.2 & NS & *** & NS \\
\hline
\end{tabular}

\footnotetext{
${ }^{a-f}$ Means in the same row with different superscripts are significantly different.
}

${ }^{*} p<0.05,{ }^{* *} p<0.01,{ }^{* * *} p<0.001$.

SEM, standard error of means; NS, not significant; $C_{2}$, Acetate; $C_{3}$, Propionate; $C_{4}$, Butyrate; $C_{5}$, Valerate; $C_{2} / C_{3}$, ratio of acetate to propionate. 


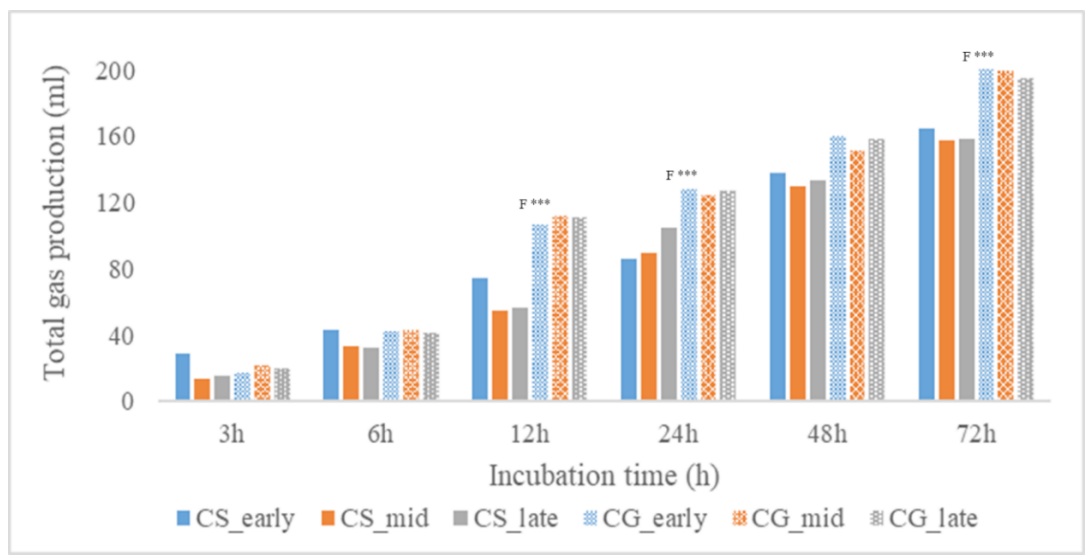

Fig. 1. Total gas production of early, mid, and late harvest corn stover and grain incubated in rumen fluid through in vitro technique. ${ }^{* *} p<0.001$. CS, corn stover; CG, corn grain; F, plant fraction.

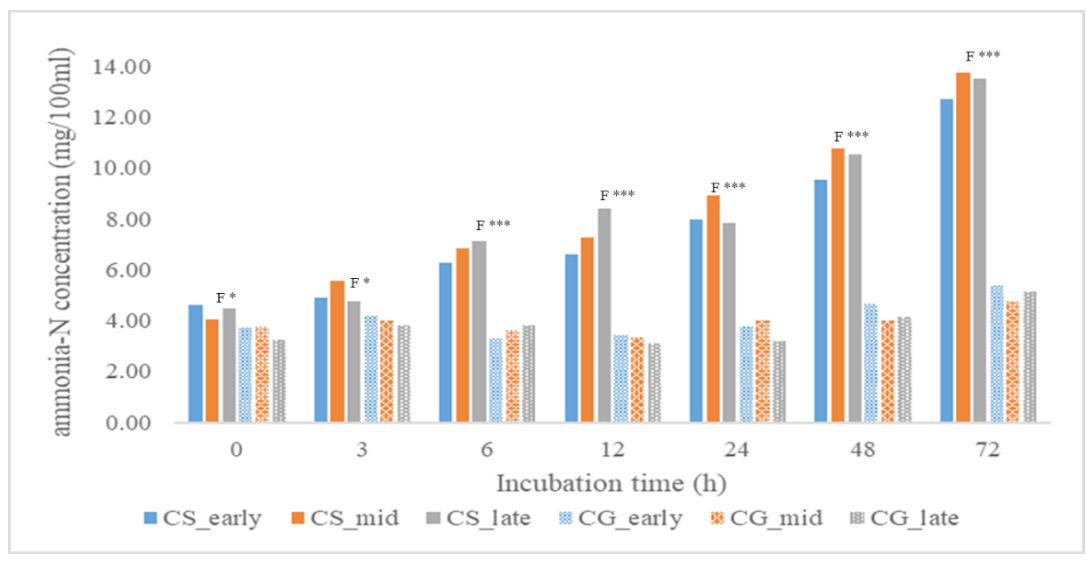

Fig. 2. The ammonia-N concentration of early, mid, and late harvest corn stover and grain incubated in rumen fluid through in vitro technique. ${ }^{*} p<0.05,{ }^{* *} p<0.001$. CS, corn stover; $\mathrm{CG}$, corn grain, F, plant fraction.

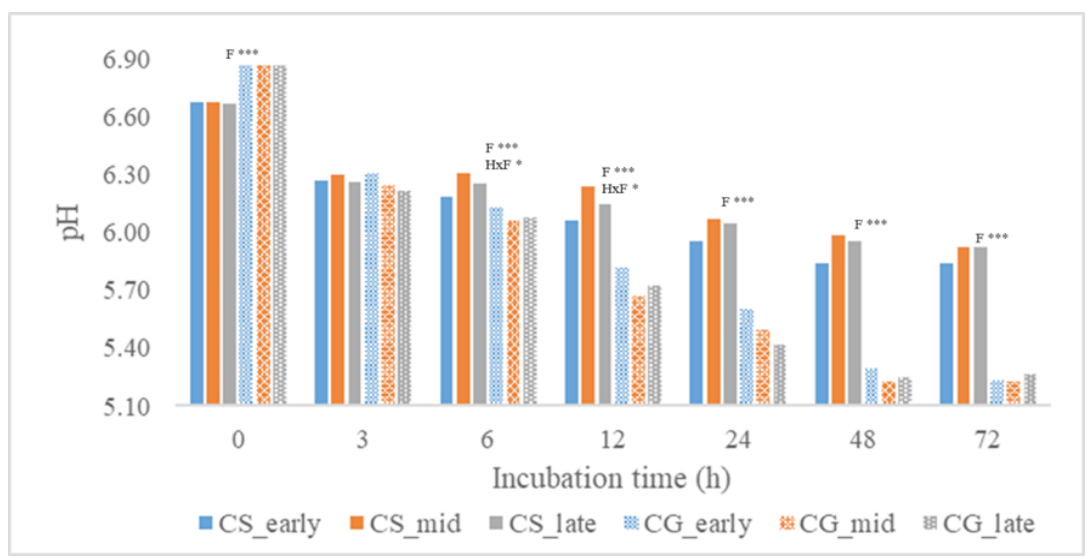

Fig. 3. pH of early, mid, and late harvest corn stover and grain incubated in rumen fluid through in vitro technique. ${ }^{*} p<0.05$, ${ }^{* *} p<0.001$. CS, corn stover; $C G$, corn grain, $F$, plant fraction, $\mathrm{H} \times \mathrm{F}$, interaction between harvest period and plant fraction.

in corn grain, and higher NDF and ADF contents were found in stover fraction. These observations were expected because as the corn plant matures, soluble carbohydrates migrate from the vege- tative fractions (stover) to ear fractions (grain) of the plant leaving the structural carbohydrates behind. As the corn plant advance in maturity, DM of the ear fraction increases due to grain filling 


\section{JAST}

and NDF decreases [14-15] due to the dilution effect as starch accumulates. Consistent with the literature cited, increased DM, and decreased NDF contents were observed in late-harvest of corn grain. The high NFC content found in the late-harvest corn grain can also be associated with dilution of fiber and increase of soluble carbohydrates during grain filling. Furthermore, late-harvest decreased $\mathrm{CP}$ and $\mathrm{EE}$ contents of corn grain although it increased the $\mathrm{ADF}$ content. For corn stover, late-harvest increased $\mathrm{DM}, \mathrm{NDF}$ and $\mathrm{ADF}$ contents and decreased $\mathrm{CP}$ content. In a number of studies, later harvest of corn plants resulted in increased DM, NDF, and ADF contents of residual parts [16] while CP content declines rapidly throughout ear maturation [17]. Some of the results in this study were found inconsistent to the literature cited. For instance, high $\mathrm{CP}$ content was observed in mid-harvest corn stover. However, it could be due to the uneven distribution of the leaves, stalks, husks, and cobs in the corn stover samples. As Li et al. [18] reported, leaves among other parts of corn stover had the highest CP content. Mid-harvest might have contained more leaves hence, the high $\mathrm{CP}$ content. Increased $\mathrm{OM}$ content in late-harvest of corn stover is associated with the high ADF content observed in the same harvest period.

Corn grain EDDM can be a good estimate to starch digestibility as proposed by Correa et al. [19] and confirmed by Ahmed et al. [20] because $70 \%$ of the total grain DM is starch, one of the grain's most digestible components. Higher EDDM found in corn grain was expected because starch is a highly fermentable carbohydrate. However, EDDM of corn grain in this study ranged only from $53 \%$ to $57 \%$ which is lower than the generally known $80 \%-90 \%$. One of the few accounts that report low DM digestibility of corn grain stated a $60.09 \%$ in vitro DM digestibility for coarsely-ground and $73.88 \%$ for finely ground corn grain [21]. EDDM values in this study are close to that of the coarsely ground which suggests that the particle size of the corn grain was not fine enough for an effective degradation. Another possible reason for the low DM digestibility of corn grain could be the diet fed to the experimental animal [22]. The Hanwoo in this study was fed $7 \mathrm{~kg}$ ryegrass and $2 \mathrm{~kg}$ concentrates. The high proportion of roughage might have increased the microbes in the rumen fluid with a preference for structural carbohydrates (fibrolytic) and limited the nonstructural carbohydrates one (amylolytic) consequently affecting the degradation of corn grain. In terms of maturity, it was reported that total DM and starch digestibility of corn grain decreased with advancing maturation [20]. Correspondingly, decreased in vitro EDDM was found in late-harvest of corn grain. The same observation was found in stover. As reported, degradability of corn stover dramatically declines with advancing maturity [23-24] due to the migration of soluble carbohydrates to ears. Late-harvest of stover led to the accumulation of cell wall contents [25] and these cell wall contents especially $\mathrm{ADF}$ and lignin contents limited the degradability of the stover. High EDNDF and EDADF were found in early-harvest regardless of plant fractions indicating that early-harvest of corn plant would provide sufficient amount of digestible cell walls available for microbial fermentation. Late-harvest stover had highest EDCP followed by mid-harvest and early-harvest of the same plant fraction. The same observation was found in the corn grain EDCP. These indicate that late-harvest resulted to easily degradable protein in vitro. However, it should be noted that $\mathrm{CP}$ degradation does not only happen in the rumen and in vitro method primarily measure DM, NDF, and ADF degradation. Thus, using in vitro method to determine EDCP could over or underestimate protein digestion in animals.

Gas production increased with the maturity of corn plants due to increased contents of fermentable cell walls in stover fraction [26], and starch in grain [20]. However, in this study, total gas production was not affected by harvest period. Despite this, a pattern was observed where harvest period with high NDF and low $\mathrm{ADF}$ contents had higher gas production. Harvest periods that showed this pattern were early-harvest of corn stover, and early and mid-harvest of corn grain. This observation suggests that these harvest periods have sufficient amount of accessible substrate for microbial fermentation that could increase gas production. While harvest period was not affected, plant fraction was significantly affected. Higher gas production in corn grain than the stover fraction can be related to its higher EDDM. As Jin et al. [27] reported, there is a strong relationship between total gas production and EDDM. The high total gas produced could have resulted from the fermentation of the easily degradable starch which represents the majority of the fermentable organic matter in corn grain. However, it should be considered that gas production could be the direct gas resulting from degradation of feeds or indirect gas from buffering of VFAs.

VFAs are the major energy source for ruminant animals. Corn grain had lower total VFA concentration than corn stover. The low total VFA concentration of corn grain could be attributed to its low EDDM. As Qin et al. [28] reported, VFA concentration is positively related to EDDM. Furthermore, the diet fed to Hanwoo might have changed the microflora in the rumen. As discussed, microbes present in the rumen fluid could be predominantly fibrolytic thus affected the EDDM and VFA production of corn grain. In addition, total VFA in this study was found close to the total VFA concentration reported by Lee et al. [21]. In the present study, total VFA of corn grain at $48 \mathrm{~h}$ ranged to 76.51 to 90.56 $\mathrm{mM}$ while Lee et al. [21] reported a total VFA of $88.61 \mathrm{mM}$ for coarsely-ground corn grain after $48 \mathrm{~h}$. As generally known, reduction of particle size of corn grain increases the surface area available for microbial attack which enhances the digestibility of feed. This 
suggest that particle size of corn grain in this study contributed to the low degradation of DM and ultimately affected the production of VFA. In terms of the harvest period, early-harvest of corn grain which showed EDDM also displayed high total VFA at 3,12, and $72 \mathrm{~h}$. On the other hand, mid-harvest stover which showed high EDDM also displayed high total VFA concentration from 12 to $72 \mathrm{~h}$. Higher concentration of VFA suggests an increased rumen microbial activity because of more available quantities of organic matter being fermented in the rumen [29].

Plant fractions influenced the molar proportions of acetate and propionate. Higher acetate proportion was observed in corn stover, and higher propionate proportion was observed in corn grain. High levels of acetate usually occur in animals fed large amounts of roughage and high levels of propionate occur to animals fed cereals because of its starch content [30]. High level of propionate in late-harvest of corn grain at 3-12 $\mathrm{h}$ can be probably due to increased starch in corn grain which also led to its low level of acetate to propionate ratio. Consistently high acetate to propionate ratio in early-harvest of stover at 3-12 $\mathrm{h}$ indicated a proportionally higher digestible NDF. Higher valerate proportion in corn grain can be associated with its higher $\mathrm{CP}$ content compared to stover. Getachew et al. [31] reported that there was a highly significant correlation between $\mathrm{CP}$ level and valerate production indicating that $\mathrm{CP}$ fermentation contributes to VFA production.

Ammonia-N concentration was not significantly affected by harvest period but was significantly affected by plant fractions at all incubation time where corn stover had higher ammonia-N concentration. The ammonia- $\mathrm{N}$ concentration of corn grain fell outside the range for the maximum efficiency of microbial synthesis. According to Sater et al. [32], the ammonia-N concentration of 5 to 8 $\mathrm{mg} \times 100 \mathrm{~mL}^{-1}$ supports maximum microbial synthesis. The observation could have resulted from catabolism of lysed cells due to the futile environment like an acidic condition. In this study, $\mathrm{pH}$ of the rumen fluid with corn grain samples had high acidity. From 12-72 $\mathrm{h}, \mathrm{pH}$ of corn grain was all below 6.0 which could be due to the accumulation of lactic acid because of starch fermentation. Weimer [33] reported that a neutral $\mathrm{pH}$ between 6 and 9 is best to ensure the growth of cellulolytic bacteria to support their digestion activity. The $\mathrm{pH}$ values of corn stover, on the other hand, were all above 6.0.

\section{Conclusions}

The best nutrient composition of corn grain was observed in early-harvest (high CP, EE, NDF, OM, NFC, and low ADF). Early-harvest of corn grain also had high EDDM, EDNDF, EDADF, and total VFA concentration. On the other hand, the best nutrient composition of stover was observed in mid-harvest (high DM, CP, $\mathrm{NDF}$, and low $\mathrm{ADF}$ ). EDDM, EDNDF, and EDADF were pro- nounced in early-harvest and mid-harvest of stover but the latter showed high total VFA concentration. Hence, early and mid-harvested corn stover and grain in a mountainous area preserved their nutrients, which led to the effective degradation of major nutrients and high VFA production. However, further study is recommended because harvest period of grain and stover did not show a systematic effect on $\mathrm{pH}$, ammonia- $\mathrm{N}$ concentration and gas production, and lactic acid concentration should be measured as well in addition to other VFA molar proportions.

\section{Competing interests}

No potential conflict of interest relevant to this article was reported.

\section{Funding sources}

This work was supported by the research grant from the Rural Development Administraion, Korea, PJ01091003 and National Research Foundation of Korea, NRF-2017RIDIA3B03034891.

\section{Acknowledgements}

Not applicable.

\section{Availability of data and materials}

Upon reasonable request, the datasets of this study can be available from the corresponding author.

\section{Authors' contributions}

Conceptualization: Choi SH, Seong HA.

Data curation: Nogoy KM, Zhang Y, Lee YH.

Formal analysis: Nogoy KM.

Methodology: Nogoy KM, Zhang Y.

Software: Nogoy KM.

Validation: Zhang Y.

Investigation: Choi SH, Seong HA.

Writing - original draft: Nogoy KM, Choi SH, Seong HA.

Writing - review \& editing: Li XZ, Choi SH, Seong HA.

\section{Ethics approval and consent to participate}

This article does not require IRB/IACUC approval because there no experiments directly conducted to human and animals.

\section{ORCID}

Kim Margarette Nogoy https://orcid.org/0000-0002-0958-7632

Yan Zhang https://orcid.org/0000-0003-2752-0578

Ye Hyun Lee

Xiang Zi Li

Hyun A Seong https://orcid.org/0000-0003-2681-8022 https://orcid.org/0000-0003-3061-3847 https://orcid.org/0000-0002-5997-9028

Seong Ho Choi https://orcid.org/0000-0001-8869-0218 


\section{References}

1. Chung KY, Lee SH, Cho SH, Kwon EG, Lee JH. Current situation and future prospects for beef production in South Korea - A review. Asian Australas J Anim Sci. 2018;31:95160.

2. Bradford GE. Contributions of animal agriculture to meeting global human food demand. Livest Prod Sci. 1999;59:95-112.

3. Andrieu J, Demarquilly C, Dardenne P, Barriere Y, Lila M, Maupetit $\mathrm{P}$, et al. Composition and nutritive value of whole maize plants fed fresh to sheep. I. Factors of variation. Ann Zootech. 1993;42:221-49.

4. Pereira MN, Von Pinho RG, Bruno RGS, Calestine GA. Ruminal degradability of hard or soft texture corn grain at three maturity stages. Sci Agric. 2004;61:358-63.

5. Silva LFP, Cassoli LD, Roma LC Jr, Rodrigues ACO, Machado PF. In situ degradability of corn stover and elephant-grass harvested at four stages of maturity. Sci Agric. 2008;65:595603.

6. Preston, TR. Tropical animal feeding: a manual for research workers. Rome (Italy): Food and Agricultural Organization of the United Nations (FAO); 1995. FAO Animal Production and Health Paper, No. 126.

7. AOAC. Official methods of analysis. 13th ed. Washington DC: Association of Official Analytical Chemists; 1995.

8. Van Soest PJ, Robertson JB, Lewis BA. Methods for dietary fiber, neutral detergent fiber, and nonstarch polysaccharides in relation to animal nutrition. J Dairy Sci. 1991;74:3583-97.

9. McDougall EI. Studies on ruminant saliva. I. The composition and output of sheep's saliva. Biochem J.1948;43:99-109.

10. Fawcett JK, Scott JE. A rapid and precise method for the determination of urea.J Clin Pathol. 1960;13:156-9.

11. Li XZ, Long RJ, Yan CG, Choi SH, Jin GL, Song MK. Rumen microbial responses in fermentation characteristics and production of CLA and methane to linoleic acid in associated with malate or fumarate. Anim Feed Sci Technol. 2010;155:132-9.

12. Orskov ER, McDonald I. The estimation of protein degradability in the rumen from incubation measurements weighted according to rate of passage.J Agric Sci. 1979;92:499-503.

13. SAS. SAS user's guide: statistics. Cary (NC): SAS Institute Inc.; 2008.

14. Filho AXS, Von Pinho RG, Pereira JLAR, dos Reis MC, de Rezende AV, Mata DC. Influence of stage of maturity on bromatological quality of corn forage. R Bras Zootec. 2011;40:1894-901.

15. Filya I. Nutritive value and aerobic stability of whole crop maize silage harvested at four stages of maturity. Anim Feed
Sci Technol. 2004;116:141-50.

16. Tolera A, Berg T, Sundstol F. The effect of variety on maize grain and crop residue yield and nutritive value of the stover. Anim Feed Sci Technol. 1999;79:165-77.

17. Johnson L, Harrison JH, Hunt C, Shinners K, Doggett CG, Sapienza D. Nutritive value of corn silage as affected by maturity and mechanical processing: a contemporary review.J Dairy Sci. 1999;82:2813-25.

18. Li HY, Xu L, Liu WJ, Fang MQ, Wang N. Assessment of the nutritive value of whole corn stover and its morphological fractions. Asian Australas J Anim Sci. 2014;27:194-200.

19. Correa CES, Shaver RD, Pereira MN, Lauer JG, Kohn K. Relationship between corn vitreousness and ruminal in situ starch degradability. J Dairy Sci. 2002;85:3008-12.

20. Ahmed S, Grecchi I, Ficuciello V, Bacciu N, Minuti A, Bani P. Effects of hybrid and maturity stage on in vitro rumen digestibility of immature corn grain. Ital J Anim Sci. 2014;13:3149.

21. Lee SY, Kim WY, Ko JY, Ha JK. Effects of corn processing on in vitro and in situ digestion of corn grain in Holstein steers. Asian Australas J Anim Sci. 2002;15:851-8.

22. Orskov ER, Hovell FDD, Mould F. The use of nylon bag technique for the evaluation of feedstuffs. Trop Anim Prod. 1980;5:195-213.

23. Berger LL, Paterson JA, Klopfenstein TJ, Britton RA. Effect of harvest date and chemical treatment on the feeding value of corn stalklage. J Anim Sci. 1979; 49:1312-6.

24. Russell JR. Influence of harvest date on the nutritive value and ensiling characteristics of maize stover. Anim Feed Sci Technol. 1986;14:11-27.

25. Kruse S, Herrmann A, Kornher A, Taube F. Evaluation of genotype and environmental variation in fibre content of silage maize using a model-assisted approach. Eur J Agron. 2008;28:210-23.

26. Van Soest PJ. Nutritional ecology of the ruminant. 2nd ed. Cornell University Press, Ithaca, New York, NY; 1994.

27. Jin GL, Shinekhuu J, Qin WZ, Kim JK, Ju JK, Suh SW, et al. Effect of protein fractionation and buffer solubility of forage sources on in vitro fermentation characteristics, degradability and gas production. J Korean Soc Grassl Forage Sci. 2012;32:59-74.

28. Qin WZ, Choi SH, Lee SU, Lee SS, Song MK. Effect of defaunation on in vitro fermentation characteristics and methane emission when incubated with forages. J Korean Soc Grassl Forage Sci. 2013;33:197-205.

29. Khandaker ZH, Steingass H, Drochner W. Supplementation of wheat straw with sesbania on voluntary intake, digestibility and ruminal fermentation in sheep. Small Rumin Res. 1998;28:23-9. 
30. Madrid J, Megias MD, Hernandez F. In vitro determination of ruminal dry matter and cell wall degradation, and production of fermentation end-products of various by-products. Anim Res. 2002;51:189-99.

31. Getachew G, Robinson PH, DePeters EJ, Taylor SJ. Relationships between chemical composition, dry matter degradation and in vitro gas production of several ruminant feeds. Anim
Feed Sci Technol. 2004;111:57-71.

32. Satter LD, Slyter LL. Effect of ammonia concentrations on rumen microbial protein production in vitro. Brit J Nutr. 1974;32:199-208.

33. Weimer PJ. Why don't ruminal bacteria digest cellulose faster? J Dairy Sci. 1996;79:1496-502. 